\title{
THE UNDERWATER TRAJECTORY BEHAVIOUR OF HEAVY OIL JET IN CROSS- FLOW FROM A BROKEN SURFACE PIPELINE
}

\author{
Portia Felix \\ Faculty of Engineering, The University of the West Indies, Trinidad \\ Email: portiafelix30@gmail.com
}

\begin{abstract}
Oil spill occurrences continue to raise varying questions and concerns about their impact on and behaviour in the marine environment as they have the potential to cause unfavourable environmental, economic and social impacts. Understanding the behaviour of oil interactions with the ocean and nearby coastal environments is crucial in maintaining a reasonable relationship between relevant stakeholders. For this purpose, oil spill numerical models are useful for predicting the movement and distribution of oil in any receiving marine environment. As a first attempt, this paper presents a very crude oil plume model to simulate the underwater behaviour of a heavy crude oil which originates from an injection point source protruding the water surface discharging heavy oil into a receiving water body of shallow water depth. Most of the existing surface oil spill models do not cater for spills that initially behave as a jet at the water surface which is an essential feature of this hypothetical oil spill scenario. The study sets a specific focus on simulating the underwater suspension and horizontal movement of an oil plume originating from a spill initially behaving as a positively buoyant jet. The model is based on a two-dimensional modified form of the classical Navier-Stokes equations and discretized using the Projection method. The model is applied in a rectangular domain with suitable boundary conditions and parameterizations to observe the underwater oil trajectory behaviour. The model is verified using a benchmark fluid flow problem and its results show reasonable relationship of specific gravity with depth. The ultimate contribution of the study can provide insights necessary for oil spill cleanup decisions as oil behaviour of this nature may pollute the underlying water.
\end{abstract}

Keywords: Underwater Oil Trajectory Behaviour, Oil Plume Model, Projection Method

https://doi.org/10.47412/KLEE9837

\section{Introduction}

A positively buoyant surface discharge oil jet can be considered to occur when a lighter or less dense, faster moving fluid is discharged downwardly into a heavier or denser, slower moving fluid, for example, heavy crude oil (lighter, faster fluid) into seawater (denser, slower fluid) ambient. In such a case, the oil is expected to rise almost immediately to the surface due to buoyancy forces. However, in a somewhat unusual manner, the oil becomes trapped at a shallow water depth and moves horizontally as shown in Fig. 1. This seemingly odd oil flow behaviour poses the question of what is the mechanism that will allow a horizontal column of heavy oil to be suspended at a shallow water depth in the water column which is not closely bounded by the seabed and has a 
small layer of water above it. Such an oil flow behaviour occurring at the water surface and behaving as a jet is is therefore of interesting concern, as it is rarely observed in water surface oil spill events, which usually behave as a surface oil slick that spreads on the surface and undergoes a series of weathering processes that is extensively studied in the literature [2].

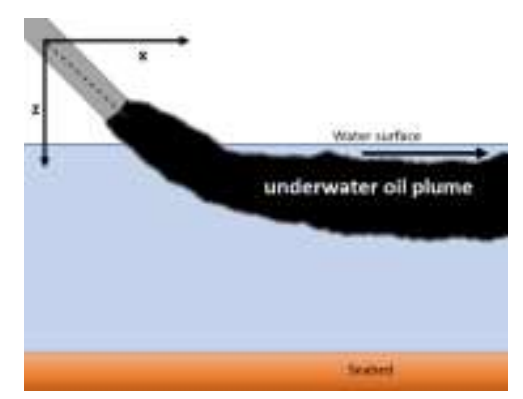

Figure 18: A positively buoyant water surface oil jet discharge

A large number of studies have been performed on the subsurface or underwater transport movement of oil originating from the water surface in which the oil forms a surface slick [1,3,8, $10,14,15,17,21,23]$. Most models subscribe to the methods of [9]. Their work considered the underwater transport of oil in the form of the dispersion process, which is the transport of suspended oil droplets from the water surface oil slick. [12 and 17] states that oil droplet size impacts buoyancy, so with oil droplets entraining in the water column from a surface slick, the larger droplets tend to the resurface while the smaller droplets remain and become dispersed in the water column due to subsurface currents oil droplet size impacts buoyancy. There are also commercially available oil spill models $[2,11,13,15,19,20]$ that track oil movement and the distribution of oil particles in the water column. All these models take into account the vertical subsurface entrainment and transport of oil as defined by [7] which states that the advection of suspended oil is the movement of oil droplets entrained in the water column due to the water current.

All of the above-mentioned studies were mathematical models based on a series of empirical algorithms that consider the underwater movement of water surface oil discharge as oil droplets. Although these models continue to produce more and more satisfactory results, the dynamics of oil rapidly plunging into the underlying water surface due to an injection source and transitioning into an underwater plume is different, mainly as the underwater oil is considered as a plume and not as droplets. Therefore, a very crude numerical approach is implemented in this present study as a first approach to provide a very basic understanding of the mechanism that can enable the suspension of the oil plume.

\section{Mathematical Model}

The mathematical formulation follows closely that of [24] and include: The two-dimensional incompressible Navier-Stokes momentum equations, the continuity equation and the energy equation. These equations can be written respectively in Cartesian form as:

Continuity equation (Conservation of mass) 


$$
\frac{\partial u}{\partial x}+\frac{\partial w}{\partial z}=0
$$

The x-momentum equation

$$
\rho_{o}\left(\frac{\partial u}{\partial t}+u \frac{\partial u}{\partial x}+w \frac{\partial u}{\partial z}\right)-\mu \nabla^{2} u+\frac{\partial p}{\partial x}=0
$$

The z-momentum equation

$$
\rho_{o}\left(\frac{\partial w}{\partial t}+u \frac{\partial w}{\partial x}+w \frac{\partial w}{\partial z}\right)-\mu \nabla^{2} w+\frac{\partial p}{\partial z}=\rho^{\prime} g
$$

The energy equation

$$
\frac{\partial S_{G}}{\partial t}+u \frac{\partial S_{G}}{\partial x}+w \frac{\partial S_{G}}{\partial z}=D\left(\frac{\partial^{2} S_{G}}{\partial x^{2}}+\frac{\partial^{2} S_{G}}{\partial z^{2}}\right)
$$

Where $t$ is the time; $\boldsymbol{x}$ and $z$ are the horizontal and vertical directions respectively; $\boldsymbol{u}$ and $\boldsymbol{w}$ are the horizontal and vertical velocity components respectively; $\rho_{o}$ is the density of the ambient fluid; $S_{G}$ is the specific gravity of the input fluid (defined as the ratio of the density of a substance to the density of a reference substance); $D$ is the diffusivity coefficient; $\rho^{\prime}$ is the deviation density; $p$ is the pressure; $g$ is the gravitational acceleration; $\mu$ is the dynamic viscosity.

Note for the energy equation, specific gravity, $S_{G}$ was used instead of temperature $T$. The mathematical rationale being: specific gravity is a function of temperature, i.e. $S_{G}=f(T)$, since $S_{G}$ is a ratio of densities (as defined above) and if the density value at the denominator is known, the density at the numerator can be solved as a function of $T$. Using the equation of state $\rho=f(T, S)$ and considering as a first approximation that the computational domain is homogeneous (constant salinity), i.e. $\rho_{o}=1$ we have:

$$
\begin{aligned}
& S_{G}=\frac{\rho(T)}{\rho_{o}} \text { where } \rho_{o} \text { is known. } \\
& \rho(T)=\rho_{o} *\left[1-\alpha\left(T-T_{o}\right)\right] \\
& \Rightarrow S_{G}=1-\alpha\left(T-T_{o}\right) \Rightarrow T=T_{o}+\frac{1-S_{G}}{\alpha}
\end{aligned}
$$


Therefore, the differential energy equation is the same, as $T$ is linear in $S_{G}$ and so $S_{G}$ is used instead of $T$.

The following dimensionless variables are used in this formulation, where $U$ is the reference velocity for the fluid system in the horizontal direction and the variables with superscript * are the dimensionless variables defined earlier:

$$
\begin{array}{llr}
x^{*}=\frac{x}{L} & z^{*}=\frac{z}{L} & t^{*}=\frac{t}{L / U} \\
p^{*}=\frac{p}{\rho_{o} U^{2}} & u^{*}=\frac{u}{U} & w^{*}=\frac{w}{U}
\end{array}
$$

Substituting the dimensionless variables into equation (1b) and dropping off the $*$ gives:

$$
\frac{\partial u}{\partial t}+u \frac{\partial u}{\partial x}+w \frac{\partial u}{\partial z}=-\frac{\partial p}{\partial z}+\frac{1}{\operatorname{Re}}\left(\frac{\partial^{2} u}{\partial x^{2}}+\frac{\partial^{2} u}{\partial z^{2}}\right)
$$

where Re is the Reynolds number defined as the ratio of the inertial forces to viscous forces within a fluid. Similarly, equation (1c) in non-dimensionalized form becomes:

$$
\frac{\partial w}{\partial t}+u \frac{\partial w}{\partial x}+w \frac{\partial w}{\partial z}-\frac{1}{\operatorname{Re}} \nabla^{2} w+\frac{1}{\rho_{o}} \frac{\partial p}{\partial z}=-\frac{g^{\prime} \rho^{\prime}}{\rho_{o}}
$$

The buoyancy force term is:

$$
f_{B}=\frac{g^{\prime} \rho^{\prime}}{\rho_{o}}
$$

It is therefore convenient to let the buoyancy force term be equal to $\operatorname{RiS}_{G}$ i.e.

$$
f_{B}=\frac{g^{\prime} \rho^{\prime}}{\rho_{o}}=R i S_{G}
$$

Where $R i$ is the dimensionless Richardson number, defined as the ratio of the buoyancy term to the flow shear term.

The same non-dimensional technique is used for both the continuity and transport equations. Therefore, by the non-dimensionalization and simplification method mentioned earlier, the following set of equations were derived and solved numerically:

$$
\begin{aligned}
& \frac{\partial u}{\partial x}+\frac{\partial w}{\partial z}=0 \\
& \frac{\partial u}{\partial t}+u \frac{\partial u}{\partial x}+u \frac{\partial w}{\partial z}=-\frac{\partial p}{\partial x}+\frac{1}{\operatorname{Re}}\left(\frac{\partial^{2} u}{\partial x^{2}}\right)+\frac{1}{\operatorname{Re}}\left(\frac{\partial^{2} u}{\partial z^{2}}\right)
\end{aligned}
$$




$$
\begin{gathered}
\frac{\partial w}{\partial t}+u \frac{\partial w}{\partial x}+w \frac{\partial w}{\partial z}=-\frac{\partial p}{\partial z}+\frac{1}{\operatorname{Re}}\left(\frac{\partial^{2} w}{\partial x^{2}}\right)+\frac{1}{\operatorname{Re}}\left(\frac{\partial^{2} w}{\partial z^{2}}\right)+R i S_{G} \\
\frac{\partial S_{G}}{\partial t}+u \frac{\partial S_{G}}{\partial x}+w \frac{\partial S_{G}}{\partial z}=\frac{\partial^{2} S_{G}}{\partial x^{2}}+\frac{\partial^{2} S_{G}}{\partial z^{2}}
\end{gathered}
$$

Where $R i$ is the Raleigh number which describes the ratio of buoyancy to viscous forces acting on the flow. Equation (6) through to equation (9) are then solved numerically by the finite difference Projection Method approximations. Brief details of this numerical analysis is explained in the following sections.

\section{Numerical Technique}

The Chorin-type projection method as described by [6] is used to solve the set of governing equations (6) to (9). The solution procedure for the Projection method is a two-step scheme involving a predictor step and a pressure corrector step respectively. In the first step an intermediate velocity field is computed using the momentum equations. This velocity however, does not satisfy the continuity equation and so in the second step a Poisson equation for the pressure is solved which is derived using the continuity equation. The intermediate velocity field is then projected onto a divergence-free velocity field using the computed pressure. A very brief outline of the steps showing the major equations to solve the governing equations are as follows:

Step 1. We begin with the predictor step in which an incomplete form of the momentum equations is integrated to yield an approximate velocity field, which will not be divergence free. That is to say, the system is advanced in time to find an intermediate flow field $U^{*}$ and $W^{*}$ using explicit Euler that satisfies the non-linear advection and diffusion terms as follows:

$$
\begin{aligned}
& \frac{U^{*}-U^{n}}{\Delta t}=-\left(\left(U^{n}\right)^{2}\right)_{x}-\left(U^{n} W^{n}\right)_{z}+\frac{1}{\operatorname{Re}}\left(U_{x x}^{* *}+U_{z z}^{* *}\right) \\
& \frac{W^{*}-W^{n}}{\Delta t}=-\left(\left(U^{n} W^{n}\right)\right)_{x}-\left(\left(W^{n}\right)^{2}\right)_{z}+\frac{1}{\operatorname{Re}}\left(W_{x x}^{* *}+W_{z z}^{* *}\right)
\end{aligned}
$$

Step 2. Now for the pressure corrector step, a projection operator (pressure term) is introduced to correct for the continuity equation, i.e. solving a Poisson equation:

$$
-\Delta p^{n+1}=-\frac{1}{\Delta t} \nabla U^{*}
$$

Computing the gradient of the pressure and the velocity field at time step $n+1$ gives:

$$
U^{n+1}=U^{*}-\Delta t \nabla p^{n+1}
$$

Similarly, this method was done for the energy equation. These steps are iterative until the solution is converged and the integration continues to the next step. 


\subsection{Spatial Discretization}

The spatial discretization is performed on a staggered grid with the pressure $p$ in the cell centres, the velocities $U$ and $W$ are placed on the midpoints of the vertical and horizontal cell boundaries respectively. An example of the staggered grid-approach structure representing the physical quantities of the problem is shown in Fig. 2.

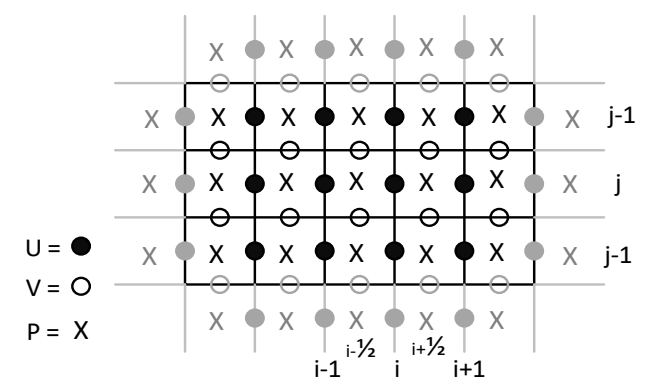

Figure 19: Staggered grid showing the spatial discretization

Where the domain boundary is indicated by the thick black line on which the boundary conditions are imposed. The dark points are the interior points while the grey points on the boundary are the boundary points and those outside the domain are the dummy or bluff points. The velocity matrices $U$ and $W$ contain only the unknown velocity values at the inner cells and not the known boundary values. At time $t=0$ the velocities are initiated with zeros (i.e. the fluid is at rest). First order derivatives are discretized with a central difference over the inner points. The boundary points in $U$ and $W$ are also included in the matrices and so the matrices are extended at the right, left, top and bottom boundaries. The central differences for the inner horizontal $u$ and vertical $w$ velocity points are defined as an example:

$$
u=\frac{u_{i+\frac{1}{2}, j}-u_{i-\frac{1}{2}, j}}{h} \text { and } w=\frac{w_{i, j+\frac{1}{2}}-w_{i, j-\frac{1}{2}}}{h}
$$

For the advection (non-linear) terms this is not the case and are calculated using the values of the velocity field on the boundaries of the control volumes which are different from the locations the discrete velocity field is defined.

\subsection{Boundary Conditions}

The boundary conditions are as follows: The Dirichlet boundary condition specifies a value at the boundary, so the horizontal velocity component $U$ on the left and right boundaries of the computational domain are directly applied as these velocity points lie on those boundaries. The same condition is applied for the vertical velocity $W$ on the top and bottom boundaries. However, the boundary conditions for $U$ on the top and bottom boundaries and $W$ on the left and right boundaries are imposed in the following manner:

$$
U_{b}=\frac{U_{\text {in }}+U_{\text {out }}}{2} \Rightarrow U_{\text {in }}=2 U_{b}-U_{\text {out }}=2 U_{b}
$$

The Neumann boundary condition specifies value for the normal derivative at the boundary point. The Neumann boundary condition specifies a zero value for the normal derivative along the boundary and this is applied in the following manner: 


$$
U_{b}=\frac{U_{\text {in }}+U_{\text {out }}}{2} \Rightarrow U_{b}=U_{\text {in }} \text { and for the pressure: } \frac{\partial p}{\partial n}=0
$$

The geometry of the problem that is simulated is shown in Fig. 3. This configuration allows for the downward movement of the oil and the horizontal movement and suspension of the oil at a depth beneath the water surface. Note that $\rho$ in the diagram is specific gravity.

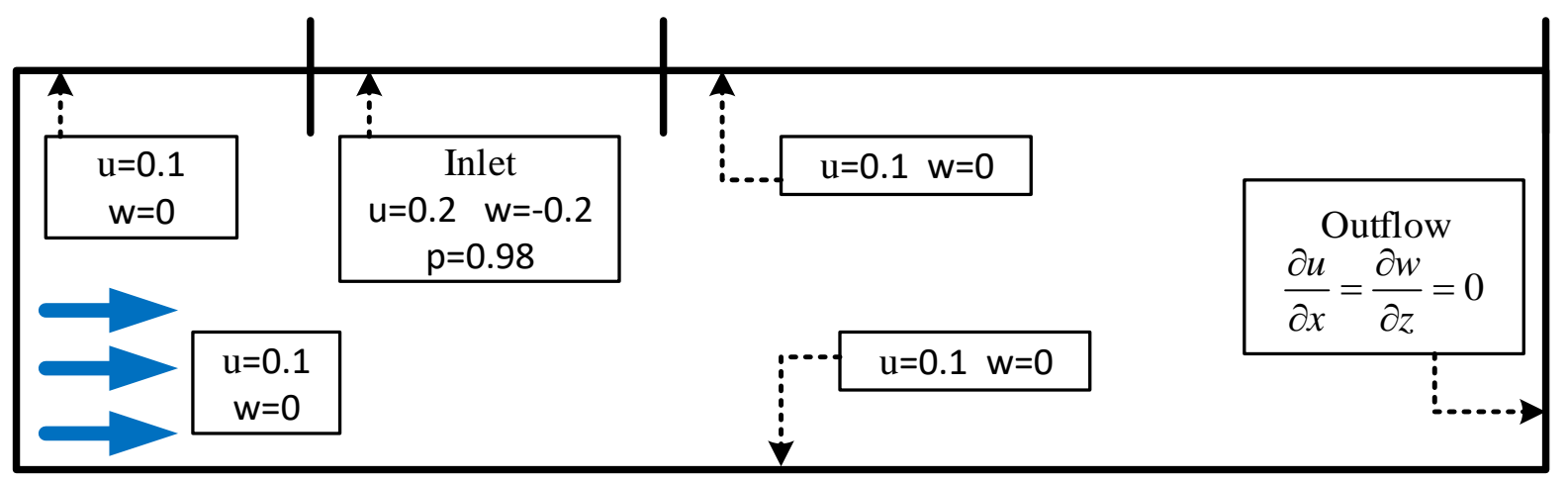

Figure 20: Geometry of the oil flow problem and the various boundary conditions

\section{Model Results}

In a first approximation, the present model has been used to simulate the suspension of the submerged oil in the water column moving along the x-direction. A simple validation of the model was first conducted by setting both the lower and upper boundaries of the computational grid to arbitrary temperatures of one degree and zero degree respectively. Therefore, density variation in the flow is generated due to the temperature gradient between the two boundaries and the density variation due to the buoyancy forces will induce the motion in the fluid. The result of this flow behaviour is observed in Fig. 4 which shows some convective plumes as expected from a temperature gradient between two plates. The model was then used to illustrate the simulation of a less dense fluid entering a slightly denser fluid. This modeling is a very simple attempt to capture the oil's lateral movement and entrapment in the water column as the fluid concentration was allowed to enter a homogeneous computational domain. Fig. 5(a) shows the computational domain in which the initial fluid injection and submergence of the less dense fluid (represented by the red colour) into the water surface of the slightly denser fluid (represented by the blue color), 10 minutes after the release. Fig. 5(b) shows the same fluid injection forming an underwater plume and floating over the denser fluid moving horizontally in the water column at 30 minutes after the release. Fig. 5(c) shows further horizontal movement and growth of the plume as it displaces more of the denser fluid. Fig. 5(d) shows the end of the model run, where it can be observed that the plume concentration appears to be suspended between the upper mixed layer and the denser blue color bottom layer forming a stable layer at a vertical depth of about 2-6 metres.

An analysis of the model results suggests that the submerged fluid travelled horizontally and became entrapped or suspended in the denser fluid at a shallow depth beneath the water surface as it appears that the submerged fluid reached a density sensitive layer with the surrounding ambient water, thereby forming a pycnocline or terminal layer. Fig. 6 shows the present model result of a plot of specific gravity versus depth. This plot illustrates the expected decreasing of specific gravity with depth as stated by $[5,18]$. The plot also shows a stable layer potentially indicating a 
pycnocline between 1-5 metres water depth, where the oil might be trapped. This may potentially verify in a very first and rough approximation, the potential capability of the present model to capture the behaviour of the oil becoming suspended.

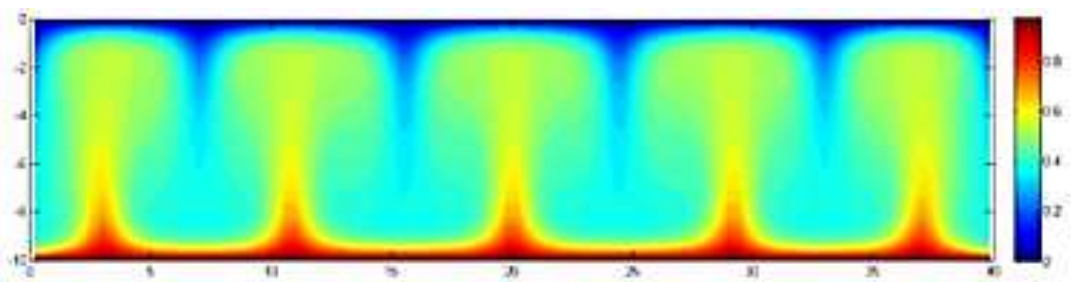

Figure 21: Benchmark model results showing flow behaviour due to temperature variation between the lower and upper boundaries
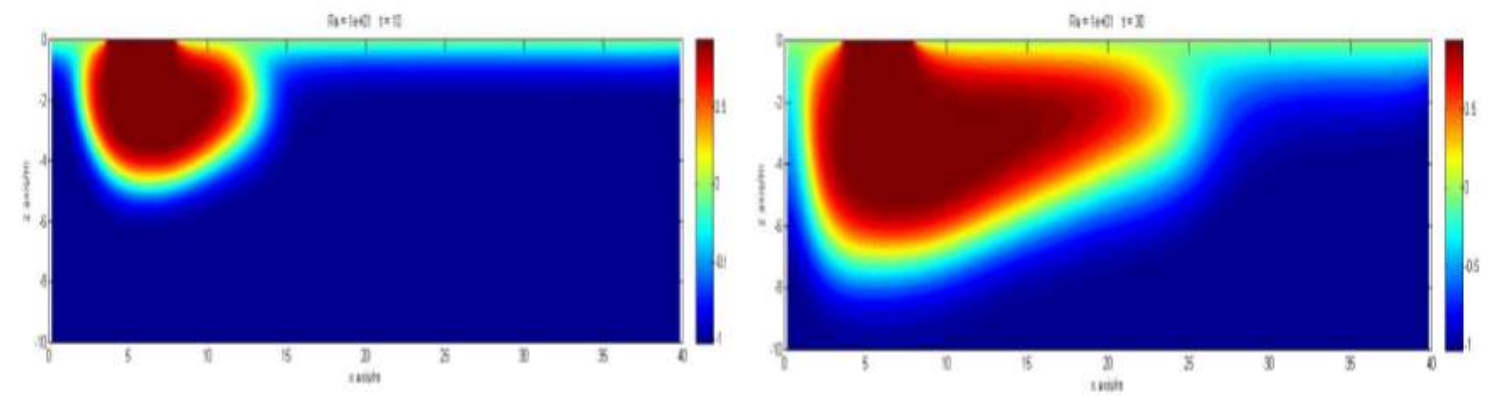

(a) Less dense fluid injected at the water surface, 10 mins after release (b) Same fluid injection, 30 mins. After release

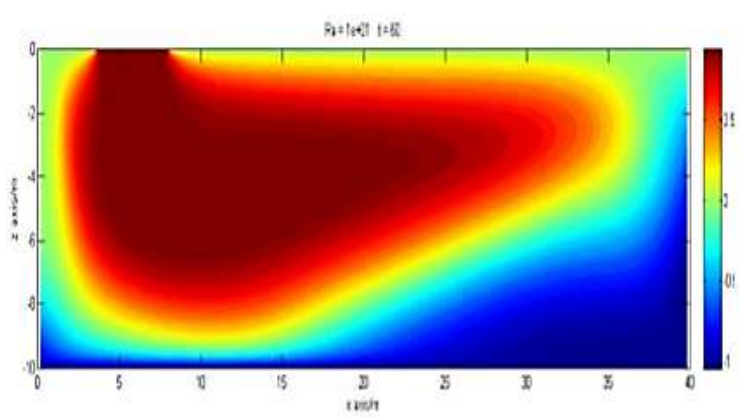

(c) Same fluid injection, 60 mins. After release After release

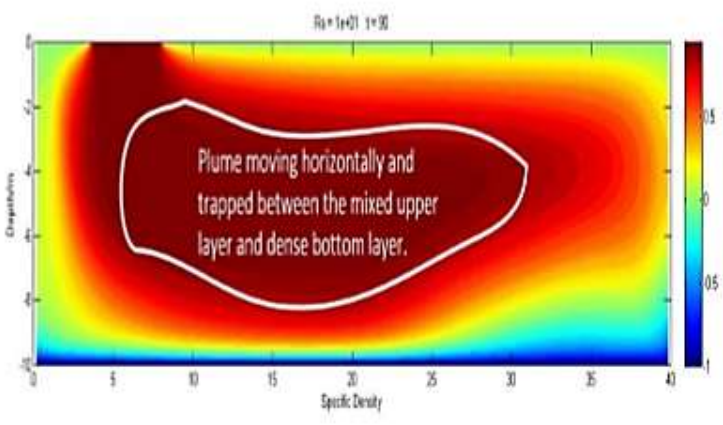

(d) Same fluid injection, 90 mins.

Figure 22: Results of present model of specific gravity (relative density) showing simulations illustrating the behaviour of a less dense (red colour) fluid discharging into a slightly more dense (blue color) ambient domain at different timesteps and remaining suspended in the water column 


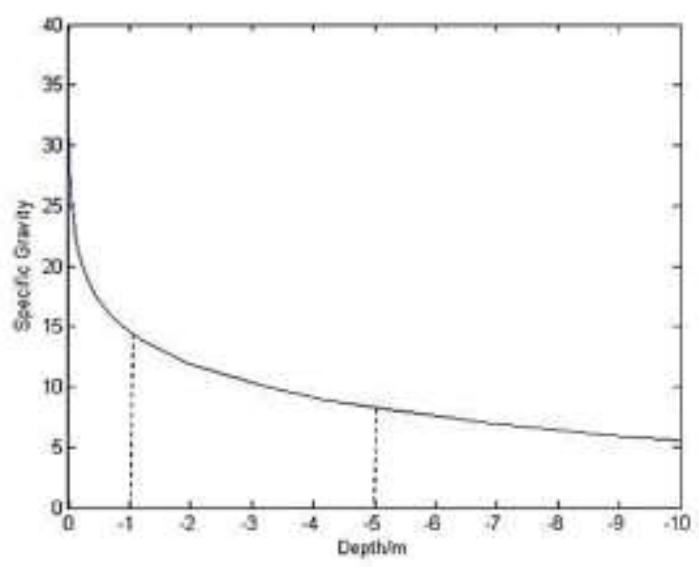

Figure 23: Present model result showing plot of specific gravity versus depth, where the distance between the dashed lines represent the stable pycnocline layer

\subsection{Results and Discussion}

In this model, the research is focused on the suspension of the oil plume at a shallow depth of $(<$ $10 \mathrm{~m}$ ) and moving downstream. The API gravity of oil is a measure of how light or heavy oil is compared to water. An API gravity value of 10 is equivalent to water, which means any oil with an API below 10 will sink while any oil with API above 10 will float. In the present study, the discharged oil is given an API value of 13.6. This feature highlights the interest of the research problem, where the oil has an API above 10, but, however, became trapped underwater instead of floating. A possible mechanism explaining the oil behaviour is that because of the high exit velocity of the initial jet (note that, the higher the exit velocity, the smaller the oil droplets i.e. spray-like), it is possible for the initial jet to mix rapidly with the slower moving ambient seawater and form smaller droplets that lose their buoyancy and begin to 'mushroom' forming an underwater oil plume at a shallow depth creating a 'terminal layer' or pycnocline. According to [5], smaller droplets increase the probability of the formation of underwater plumes. The computational model domain and input data is therefore simplified as follows: for the sake of computational expense and simplicity, it is convenient to observe the oil behaviour at more reasonable ambient and oil spill conditions. The conversion from API gravity to density first requires conversion to specific gravity $\left(S_{G}\right)$ which is given by: $S_{G}=141.5 \mathrm{API}+131.5$ and so as a first approximation to the problem, the calculated $S_{G}$ of 0.98 was used as the input oil density. A horizontal ambient velocity and smaller oil exit velocity of $0.1 \mathrm{~m} / \mathrm{s}$ and $0.2 \mathrm{~m} / \mathrm{s}$ were chosen respectively. The computational grid is a uniform rectangular grid with dimensions of $10 \mathrm{~m}$ in the vertical $\mathrm{z}$ direction and $40 \mathrm{~m}$ in the horizontal $\mathrm{x}$ direction and a mesh size of $100 \times 100$ in both directions. The total time taken for model run $=1$ hour and 40 minutes and the width of timestep $=6$ seconds. 


\section{Conclusion}

This study sets a specific focus on the underwater suspension of the oil. In which case a very simplified first step approach of a two dimensional CFD numerical model is developed to simulate the horizontal motion and entrapment of a plume-like oil mass in the water column. The model is based on the classical equations for fluid motion including: The Continuity equation, the NavierStokes momentum equations and scalar energy equation using the finite difference Projection method approach where velocity, pressure and relative density (specific gravity) fields are resolved. The results of the model (Fig. 6) appear to reproduce the general profile behaviour supporting the oil behaviour, as it is shown that in the vicinity of about 2-6m below the water surface, there is a stable layer where it appears that the oil may have become suspended and this is represented in the present model by the encircled area showing the thick dark red patch.

The model results were analysed qualitatively and showed reasonable agreement with the expected relationship between relative density (specific gravity) and depth as is described in literature, with the data also showing a stable pycnocline layer forming at a water depth of about $1-5 \mathrm{~m}$. This may verify the potential capability of the present model to capture the suspension of the oil as shown in fig. $5 \mathrm{~d}$ which shows the dense fluid trapped at a depth of about $2-6 \mathrm{~m}$ and appears to be moving horizontally. The present model results therefore illustrate as a first attempt and rough approximation, the potential capabilities of the model to simulate and study the behaviour of oil movement and neutral buoyancy beneath the water surface, and suggest that neutral buoyancy can be achieved when the effluent reaches an important sensitive density balance layer with the ambient fluid putting it in suspension in the water column. In order to see a more detailed appropriate behaviour of the underwater oil plume, more effort must be put into developing the density field to more properly define the pycnocline level where the oil was trapped and moved horizontally and using actual data of the spill event and the receiving marine ambient environment. Oils that remain suspended in the water column pose risks to certain resources that are not normally affected by floating oils [4] and so the model presented here, has the potential for becoming more appropriate in informing and aiding in cleanup decisions in such instances where the oil becomes submerged. 


\section{References}

[1]Aghajanloo,K.andPirooz,M.D.(2011)."thesimulationoftheoilweatheringprocessesinmarine environment." Proceeding of the 4th International Conference on Environmental and Computer Science, ICECS.

[2]Berry, A., Dabrowski, T., and Lyons, K. (2012). "The oil spill model oiltrans and its application to the celtic sea." Marine pollution bulletin, 64(11), 2489-2501.

[3]Betancourt, F., Palacio, A., and Rodriguez, A. (2005). "Effects of the mass transfer process in oil spill." American Journal of Applied Sciences, 2(5), 939-946. [2]

[4]Board, M. (1999). "Spills of nonfloating oils.

[5]Chopra, S., Lines, L.R.,Schmitt, D.R., and Batzle,M.L.(2010). "Heavy oils: reservoir characterization and production monitoring. Society of Exploration Geophysicists".

[6]Chorin, A. J. (1968). "Numerical solution of the navier-stokes equations." Mathematics of computation, 22(104), 745-762.

[7]Committee, A.T.etal.(1996)."State-of-the-artreviewofmodelingtransportandfateofoilspills." Journal of Hydraulic Engineering, 122(11), 594-609.

[8]De Dominicis, M., Pinardi, N., Zodiatis, G., and Lardner, R. (2013). "Medslik-ii, a lagrangian marine surface oil spill model for short-term forecasting." Geoscientific Model Development, 6(6), 1851.

[9]Delvigne,G.A.L.andSweeney,C.(1988).“Naturaldispersionofoil.”OilandChemicalPollution, 4(4), 281-310.

[10]Dunnewind, B., Bos, M., and Koops, W. (2003). "Entrainment of oil from oil spills into the water column: A new theory." International Oil Spill Conference, Vol. 2003, American Petroleum Institute, 1059-1066.

[11]Howlett,E.,Jayko,K., and Spaulding,M.(1993).“Interfacingreal-timeinformationwithoilmap.” Report no.

[12]Huang, J. C. (1983). "A review of the state-of-the-art of oil spill fate/behavior models." International Oil Spill Conference, Vol. 1983, American Petroleum Institute, 313-322.

[13]Kolluru, V., [13]Spaulding, M., and Anderson, E. (1994). "A three dimensional subsurface oil dispersion model using a particle based approach." ARCTIC AND MARINE OILSPILL PROGRAM TECHNICAL SEMINAR, MINISTRY OF SUPPLY AND SERVICES, CANADA, 867-867.

[14]Korotenko, K. A., Bowman, M. J., and Dietrich, D. E. (2010). "High-resolution numerical model forpredictingthetransportanddispersalofoilspilledintheblacksea..”Terrestrial,Atmospheric \& Oceanic Sciences, 21(1).

[15]Lehr, W., Jones, R., Evans, M., Simecek-Beatty, D., and Overstreet, R. (2002). "Revisions of the adios oil spill model." Environmental Modelling \& Software, 17(2), 189-197.

[16]Lellouche, J. m., Legalloudec, O., Regnier, C., Levier, B., Greiner, E., and Drevillon, M. (2016). "Quality information document for global sea physical analysis and forecasting product." GLOBAL"ANALYSIS" FORECAST"PHY"00, 1.

[17]Papadimitrakis, J., Psaltaki, M., Christolis, M., and Markatos, N. (2005). “Three-dimensional oil spill modelling for coastal waters." Journal of Marine Environmental Engineering, 7(4), 249260.

[18]Price,L.C.(1980).“Crudeoildegradationasanexplanationofthedepthrule.”Chemicalgeology, 28, 1-30. 
[19]Reed, M., Ekrol, N., Daling, P., Johansen, Ø., Ditlevsen, M. K., Swahn, I., Myrhaug Resby, J., and Skognes, K. (2004). "Sintef oil weathering model user's manual version 3.0.

[20]Reed, M., Gundlach, E., and [13]Kana, T. (1989). "A coastal zone oil spill model: development and sensitivity studies." Oil and Chemical Pollution, 5(6), 411-449. [21]Villoria,C.,Anselmi,A.,Garcia,F.,etal.(1991).“Anoilspillfatemodelincludingsinkingeffect.”

SPE Health, Safety and Environment in Oil and Gas Exploration and Production Conference, Society of Petroleum Engineers.

[22]Yapa, P. D., Wimalaratne, M. R., Dissanayake, A. L., and DeGraff, J. A. (2012). "How does oil and gas behave when released in deepwater?." Journal of Hydro-Environment Research, 6(4), $275-285$.

[23]Zhi-wei, L., Shu-Shen, Z., and Mead, C. (2000). "Modelling of the behavior of marine oil spills: applications based on random walk techniques." Journal of Environmental Sciences, 12(1), $1-6$.

[24] https://www.kth.se/social/upload/4f529153f276543712000001/project.pdf. 